\title{
Numerical Investigation of the Hub-Seal Mass Flow Rate Effect on the Axial Turbine Stage Efficiency
}

\author{
Petr Straka ${ }^{1, *}$ \\ ${ }^{1}$ Czech Aerospace Research Centre, Beranových 130, 19905 Prague, Czech Republic
}

\begin{abstract}
The contribution deals with numerical simulation of compressible flow through the axial turbine stage equipped with the hub-seal. The current flowing from the hub-seal has a major impact on the secondary flow in the hub-region of the blade span. The aim of this work is to found a dependency of the efficiency-drop on the hub-seal mass flow rate. Numerical simulation has been made for configuration of experimental axial single-stage reaction turbine.
\end{abstract}

\section{Introduction}

One of the significant effects, that influences flow in peripheral parts of the blade-span, is the outlet stream from the labyrinth-seal (hub-seal at the root part $[1,2]$ or shroud seal at the tip part [3]). This secondary stream has important impact on the efficiency drop in the axial turbine stages. This paper deals with numerical simulation of the hub-seal leakage flow effect on the axial turbine stage efficiency.

Figure 1 shows the scheme of the simplified axial turbine stage. Simplification lies in the absence of the rotor blade shroud. Next simplification is disconnection of the inlet to the stage and inlet to the hub-seal. Hub seal containing two seal-fins has its own inlet boundary. The mass flow rate through the hub-seal is controlled with the size of the radial clearance $c_{\text {rad }}$ in range from
$0.1 \mathrm{~mm}$ up to $1.5 \mathrm{~mm}$. Hub diameter of the axial turbine stage is $400 \mathrm{~mm}$, the blade span is $45.5 \mathrm{~mm}$. The blade span to the mean blade chord ratio is approx. 1.67. Two axial clearances $c_{a x}=5 \mathrm{~mm}$ and $10 \mathrm{~mm}$ were tested. The inlet boundary to the axial turbine stage lies in distance of $42 \mathrm{~mm}$ before the leading edge of the stator blade at the middle diameter, the outlet boundary lies in distance of $42 \mathrm{~mm}$ behind the trailing edge of the rotor blade at the middle diameter.

The isentropic outlet Mach number is $M_{i s, \text { out }}=0.24$, the isentropic outlet Reynolds number is $R e_{i s, \text { out }} \approx$ $5.1 \times 10^{6}$, rotational speed of the rotor blades is 3810 RPM. The flowing medium is perfect gas of the heats capacity ratio $\gamma=1.2229$ and the specific gas constant $R$ $=407.86 \mathrm{~J} \cdot \mathrm{kg}^{-1} \cdot \mathrm{K}^{-1}$.

Original count of blades is 78 stator and 56 rotor blades. This count was modified for numerical

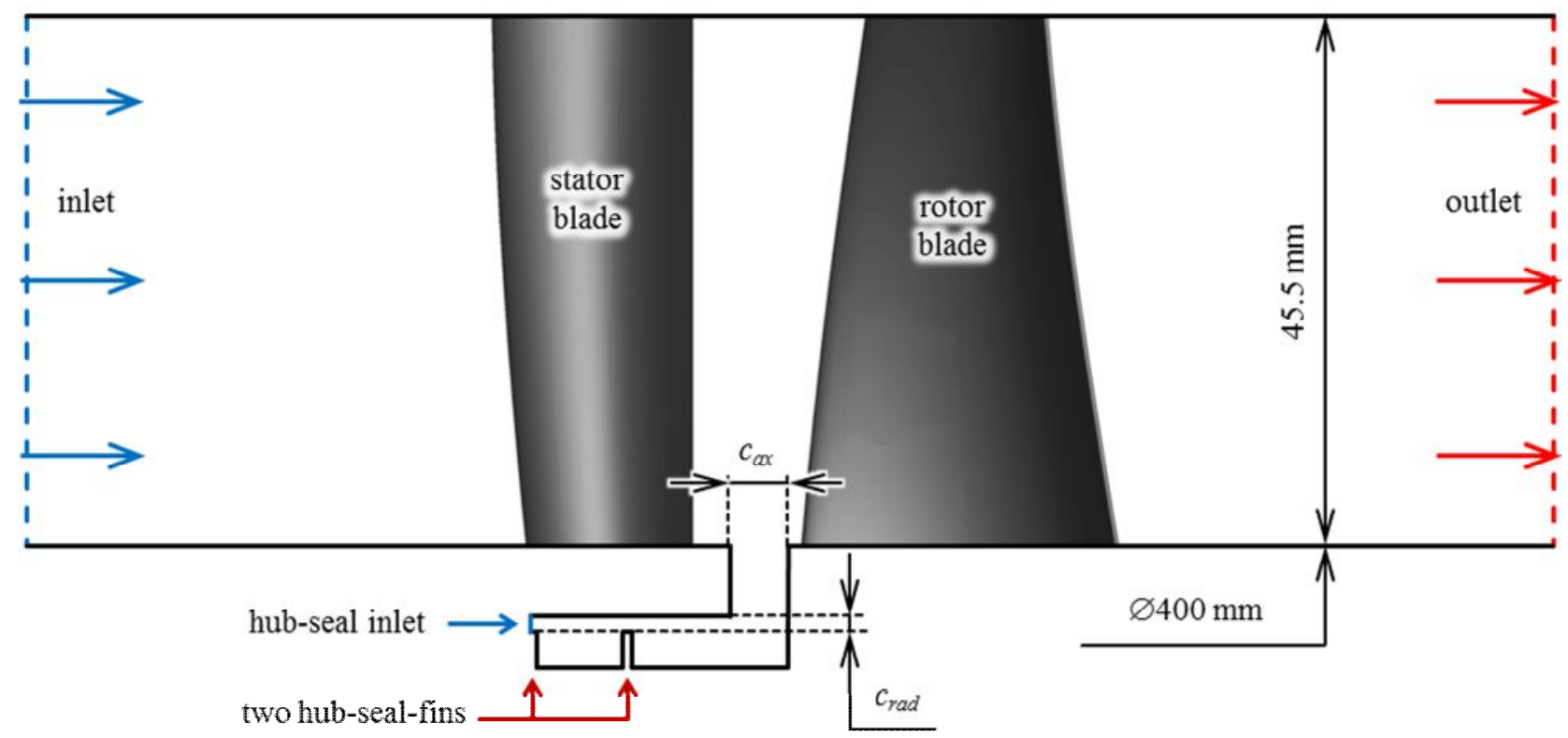

Fig. 1. Scheme of the axial turbine stage with the hub-seal. 


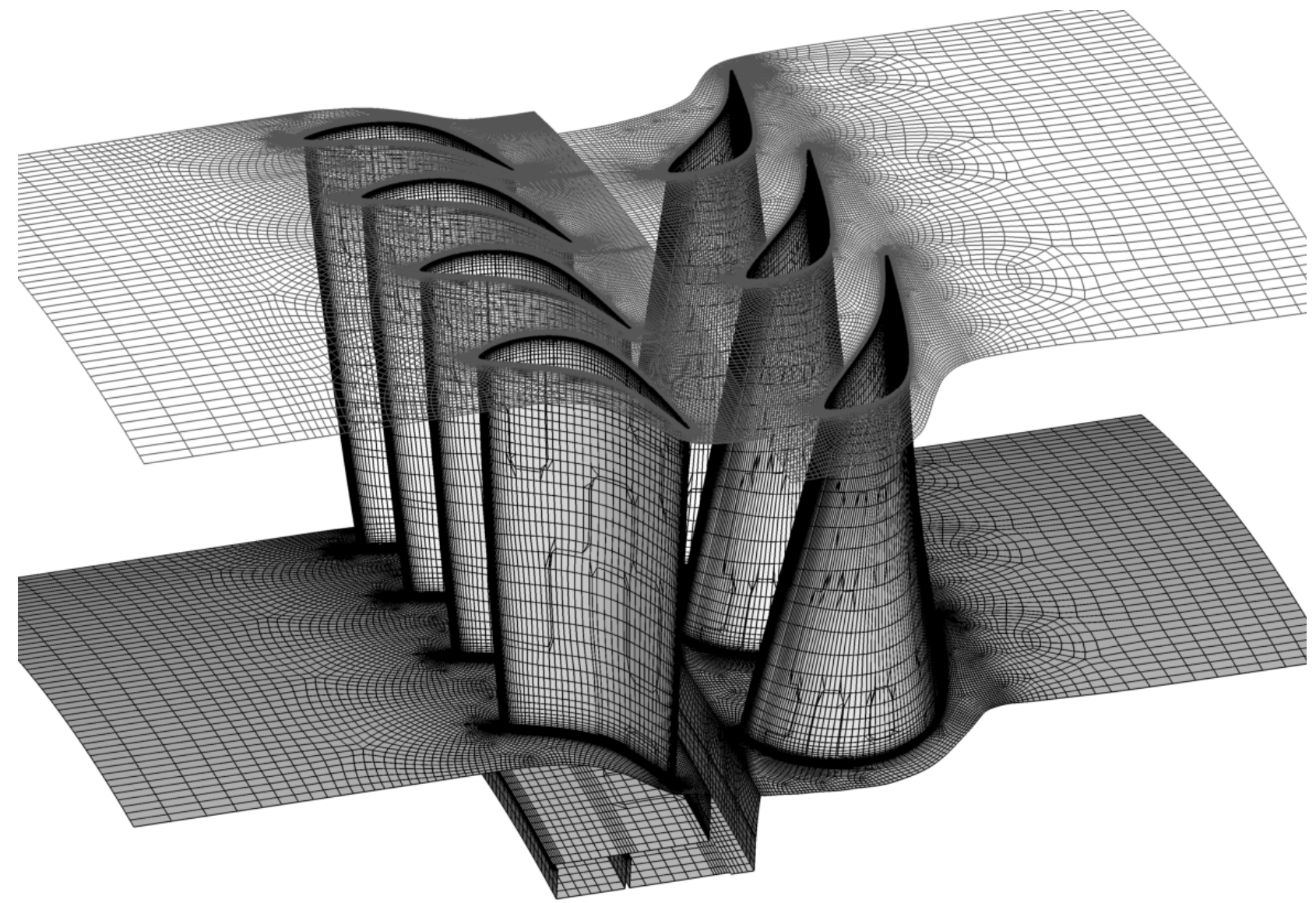

Fig. 2. Computational mesh.

simulation in order to decrease the computational performance requirements, as will be discussed later.

Calculations of flow were performed using the inhouse numerical software, which is based on solution of RANS equations closed with two-equations turbulence model (for more details see $[4,5,6]$ ). The nonlinear explicit algebraic model of the Reynolds stress is used in this work [7]. Figure 2 shows multi-block structured computational mesh of hexahedral cells with almost $9.1 \times 10^{6}$ nodes. The computational mesh is refined close to the walls to ensure that the viscous sub-layer is covered at least by five cells.

At the inlet boundaries (to the axial stage as well as to the hub-seal) there were prescribed the total temperature $T_{T 0}=598.107 \mathrm{~K}$, the total pressure $p_{T 0}=$ $5.005677 \mathrm{MPa}$, the inlet turbulence intensity $T u_{0}=2.5 \%$, ratio of the turbulent and the molecular viscosity $\mu_{\tau} / \mu=$ 100 and axial flow direction. At the outlet boundary

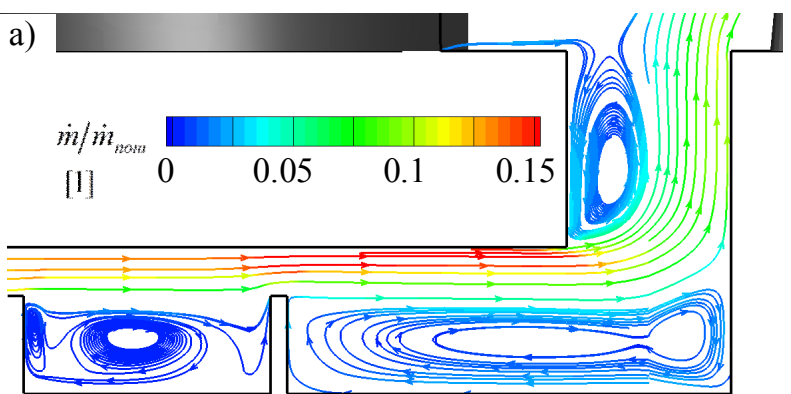

there was prescribed the static pressure at the hub diameter $p_{S 2}=4.828995 \mathrm{MPa}$ together with the radial equilibrium assumption. On all solid walls there was prescribed no-slip condition together with adiabatic condition (no heat transfer through the wall).

In order to reduce the computational cost original number of blades (78 stator and 56 rotor blades) was modified. The blade geometry was slightly scaled in the circumferential section, not in radial direction. The computational domain (shown in figure 2) covers periodically repeated section containing four stator and three rotor blades, where the scale factor of stator blades is $i_{s}=1.022$ and the scale factor of rotor blades is $i_{r}=$ 0.978. Note that scaling has no effect on static aerodynamics loading of the blades, because the pitch to chord ratio of blades is preserved. Scaling of the blade profile causes little change of the chord based Reynolds number, but it is minor problem in case of the scale

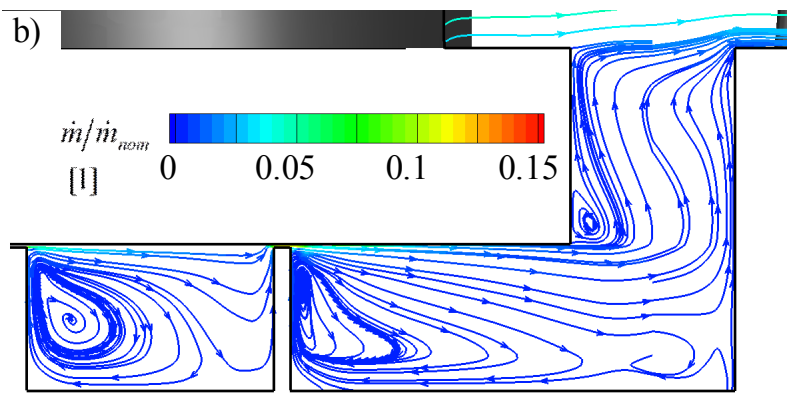

Fig. 3. Projection of streamlines to the meridian plane in the hub-seal region; the streamlines are coloured by normalized mass-flux density; radial clearance: a) $c_{r a d}=1.5 \mathrm{~mm}$, b) $c_{\text {rad }}=0.1 \mathrm{~mm}$. 
factors near value 1. The method of scaling has, however, major impact on prediction of dynamic aerodynamic loading of the blades. Therefore this method is unusable in cases where such that prediction is a main scope. On the other hand, prediction of secondary flows effect is only slightly affected by scaling of blade geometry when the scale factor is not far from value 1 (as discussed e.g. in $[8,9]$ ).

The interface between stator and rotor part of the computational domain is performed via the sliding mesh method, where communication between stationary stator domain and rotating rotor domain is based on interpolation.

\section{Numerical results}

The solution algorithm was set for unsteady flow second order accuracy in time and space. The time step of simulation $\Delta t$ is linked with the rotational step $\Delta \varphi$ of the rotor part through the angular velocity. Number of the rotational steps $\Delta \varphi$ per periodically repeated section of four stator and three rotor blades was set to $\varphi_{\text {period }} / \Delta \varphi$ $=408$, it means approx. hundred steps for passing one pitch of the stator blade. After the initial condition disappears the flow field was averaged during three periods. The averaged flow field was then used for evaluation of the mass flux $q_{\text {seal }}$ through the inlet boundary to the hub-seal, for evaluation of total mass flux $q_{\text {tot }}$ through the outlet boundary and for evaluation the efficiency drop in axial section behind the rotor blades.

Figure 3 shows comparison of streamlines projection to the meridian plane for cases with maximal $(1.5 \mathrm{~mm})$ and minimal $(0.1 \mathrm{~mm})$ radial clearance $c_{\text {rad }}$ above the seal-fins. The streamlines are coloured with the normalized mass-flux density $\dot{m} / \dot{m}_{\text {nom }}$ defined as

$$
\dot{m}=\sqrt{\left(\rho u_{a x}\right)^{2}+\left(\rho u_{r a d}\right)^{2}},
$$

where $\rho$ is the density, $u_{a x}$ and $u_{\text {rad }}$ are axial and radial velocity vector components, $\dot{m}_{n o m}$ is nominal value for normalization.

In figure 4 there are shown (looking on the rotor

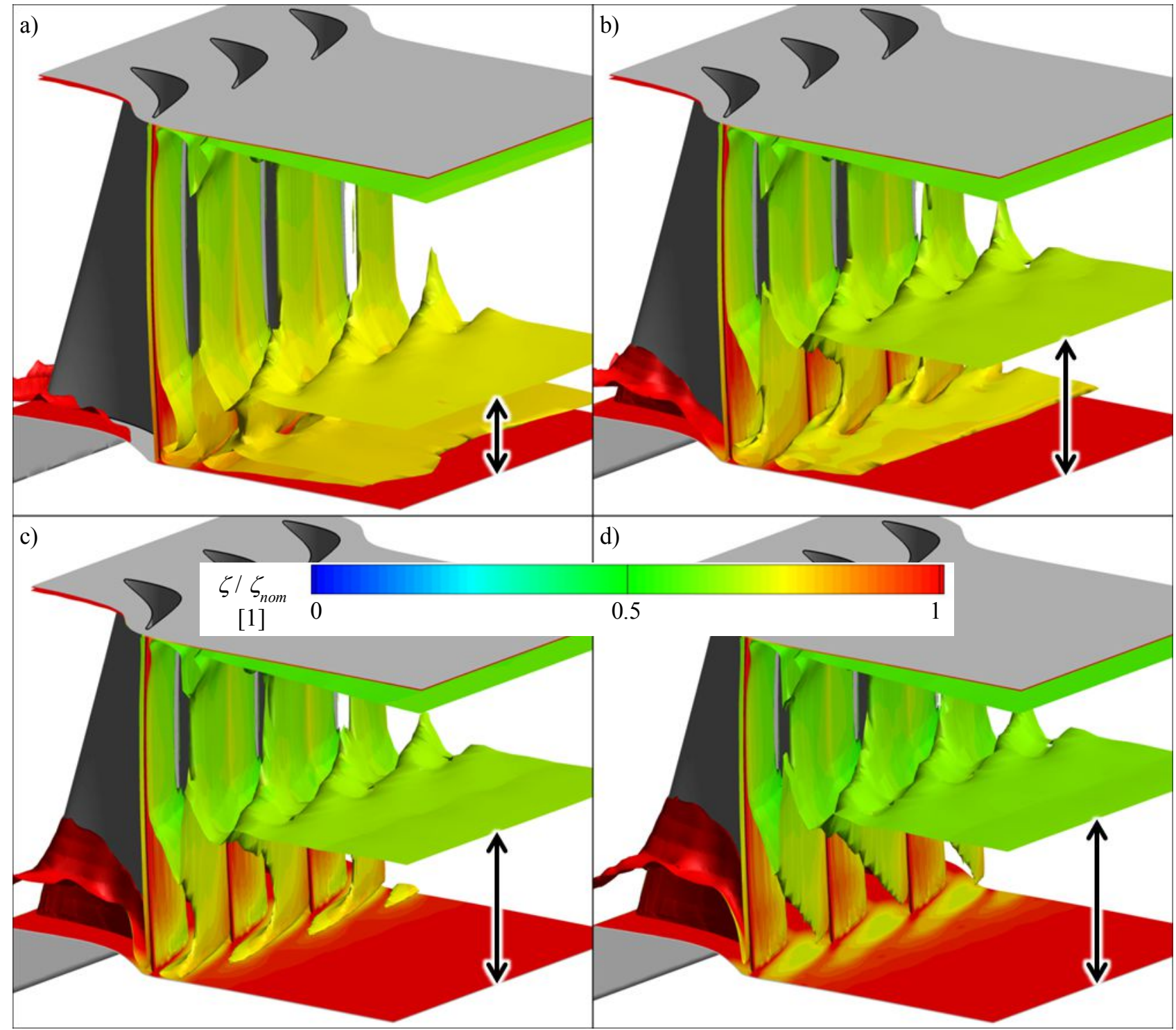

Fig. 4. Isosurface of the normalized entropy index coloured by local normalized kinetic energy loss coefficient for radial clearances $0.1 \mathrm{~mm}(\mathrm{a}), 0.5 \mathrm{~mm}(\mathrm{~b}), 1.0 \mathrm{~mm}(\mathrm{c})$ and $1.5 \mathrm{~mm}(\mathrm{~d})$; shown in cylindrical coordinates. 

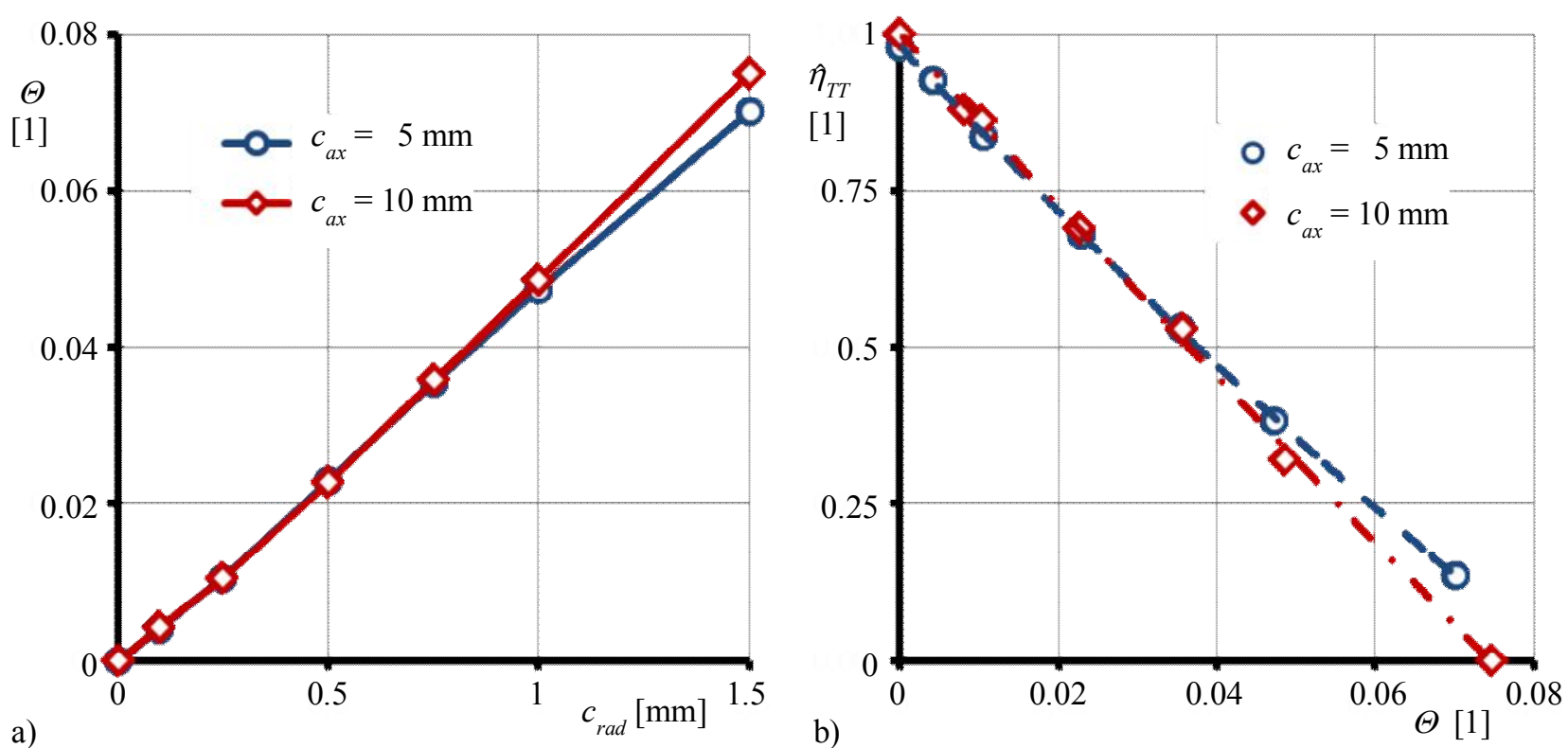

Fig. 5. a) dependency of the hub-seal mass-flux to total mass-flux ratio on the radial clearance; b) dependency of the normalized efficiency on the hub-seal mass-flux to total mass-flux ratio.

blade trailing edges) isosurfaces of normalized entropy index $s / s_{\text {nom }}$ coloured with normalized local kinetic energy loss coefficient $\zeta / \zeta_{\text {nom }}$. The entropy index and the local kinetic energy loss coefficient are defined as

$$
\begin{gathered}
s=\frac{p}{\rho^{\gamma}}, \\
\zeta=1-\frac{u_{r e l}^{2}}{u_{i s}^{2}},
\end{gathered}
$$

where $p$ is the pressure, $\rho$ is the density, $\gamma$ is the heats capacity ratio, $u_{r e l}$ is local relative velocity magnitude, $u_{i s}$ is local isentropic velocity and $s_{\text {nom }}$ and $\zeta_{\text {nom }}$ are nominal values for normalization. Because the entropy index displays dissipative areas we can see in figure 4 how much of the rotor blade span is affected by interaction of stream from the hub-seal with the rotor blades. For better clarity flow fields in figure 4 are depicted in cylindrical coordinates. We can see that in case of minimal radial clearance $c_{\text {rad }}=0.1 \mathrm{~mm}$ lower quarter of the blade span is affected whereas in case of maximal clearance $c_{\text {rad }}=$ $1.5 \mathrm{~mm}$ it is full lower half of the blade span. Note that in figure 4 there are shown not averaged but instantaneous flow fields.

Dependency of the mass-flux ratio $\Theta=q_{\text {seal }} / q_{\text {tot }}$ on size of the radial clearance is shown in figure $5 \mathrm{a}$. We can see that this dependency is nearly linear (which corresponds with $[1,2]$ ) and that the mass flow rate through the hub-seal with radial clearance $c_{\text {rad }}=1.5 \mathrm{~mm}$ is little bit higher in case of axial clearance $c_{a x}=10 \mathrm{~mm}$. This is given by different formation of radial stream behind the last seal-fin as shown in figure 6. Steeper turning of stream to the radial direction in case of $c_{a x}=5$ $\mathrm{mm}$ is more dissipative than in case of $c_{a x}=10 \mathrm{~mm}$.

Figure $5 b$ shows dependency of the normalized efficiency $\hat{\eta}_{T T}$ on the mass-flux ratio $\Theta=q_{\text {seal }} / q_{\text {tot }}$. The normalized efficiency drop is defined as

$$
\begin{gathered}
\hat{\eta}_{T T}=\frac{\eta_{T T}-\eta_{\min }}{\eta_{\max }-\eta_{\min }}, \\
\eta_{T T}=\frac{T_{T 0}-T_{T}}{T_{T 0}-T_{T i s}},
\end{gathered}
$$

where $T_{T 0}$ is the total inlet temperature, $T_{T}$ is local total temperature, $T_{T i s}$ is local isentropic total temperature, $\eta_{\min }$ and $\eta_{\max }$ are chosen minimal and maximal values for normalization. We can see that for higher value of the mass-flux ratio $\Theta$ (radial clearances 1 and $1.5 \mathrm{~mm}$ ) the case of axial clearance $c_{a x}=10 \mathrm{~mm}$ is affected by a larger decrease of the efficiency.

Distribution of normalized efficiency in axial section behind the rotor blades is shown in figure 7. This figure

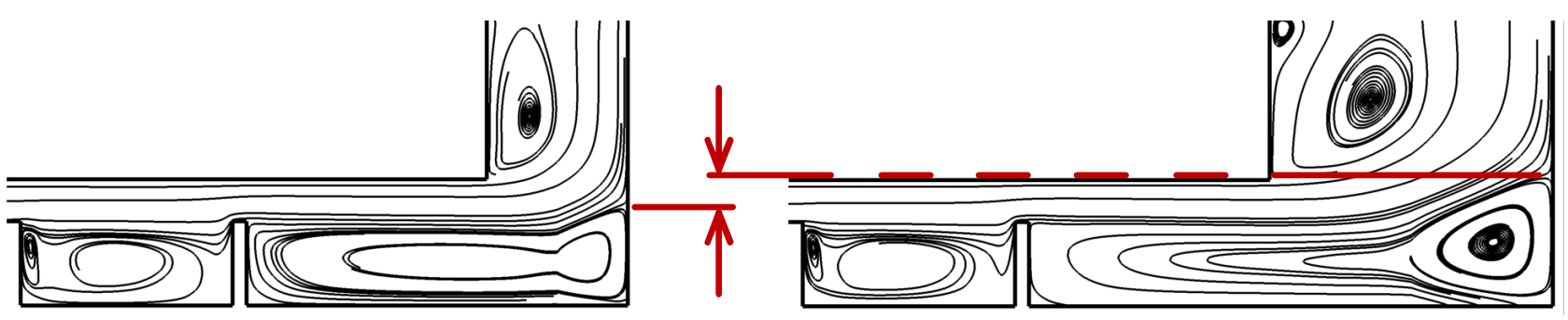

Fig. 6. Comparison of streamlines projection to the meridian plane for cases of axial clearance $c_{a x}=5 \mathrm{~mm}$ (left) and $c_{a x}=10 \mathrm{~mm}$ (right); both with maximal radial clearance $c_{r a d}=1.5 \mathrm{~mm}$. 


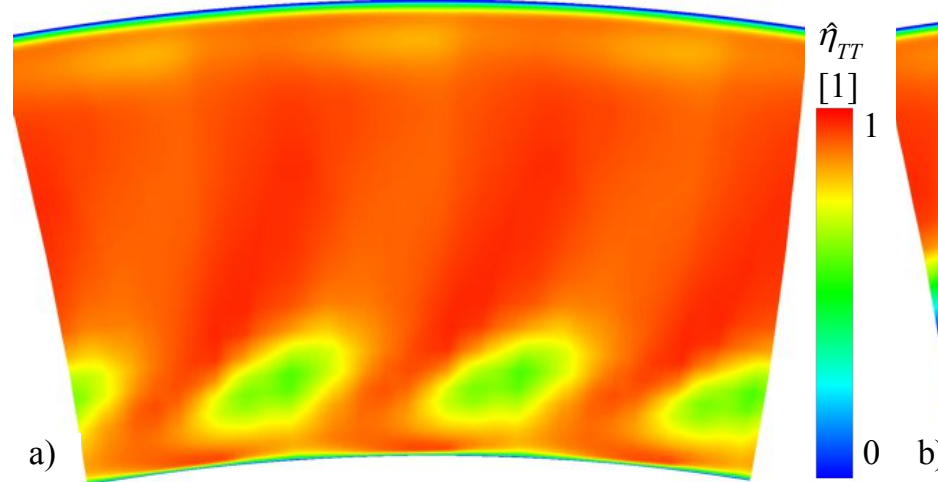

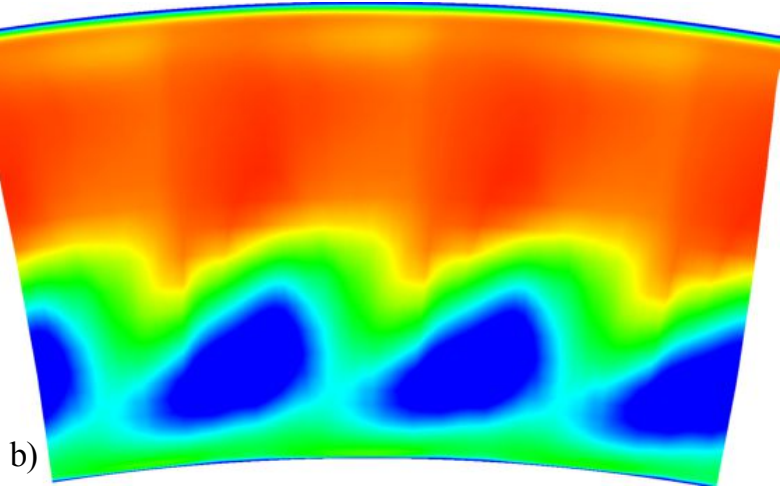

Fig. 7. Distribution of the normalized efficiency in axial section behind the rotor blades; radial clearance: a) $\left.c_{\text {rad }}=0.1 \mathrm{~mm}, \mathrm{~b}\right) c_{\mathrm{rad}}=$ $1.5 \mathrm{~mm}$.

illustrates significant difference in affected area by the interaction of stream from the hub-seal with the rotor blades for cases of minimal $(0.1 \mathrm{~mm})$ and maximal $(1.5$ $\mathrm{mm}$ ) radial clearance.

\section{Conclusions}

The interaction of stream from the hub-seal with the rotor blade was studied using numerical simulation on simplified model of the axial turbine stage containing four stator and three rotor blades and the hub-seal with two seal-fins and with separated inlet boundary. The main aim of this work was to found the dependency of the efficiency drop on the mass flow rate through the hub-seal. Knowledge of this dependency is particularly valuable for design process of new axial turbine stages. Results has shown that mass flow rate through the hubseal around seven percent of the total mass flux is able to knock down the efficiency of the stage in lower half of the blade span.

This work has been supported by the project TH02020086 of the Technology Agency of the Czech Republic.

\section{References}

1. P. Straka, EPJ Web of Conferences 180, 02100 (2018)

2. P. Straka, J. Pelant, Proc. Conf. Engineering Mechanics 2018, Svratka, pp. 821-824 (2018)

3. P. Straka, J. Pelant, M. Němec, T. Jelínek, P. Milčák, EPJ Web of Conferences 143, 02117 (2017)

4. P. Straka, EPJ Web of conferences, 25, 01090 (2012)

5. P. Straka, Report VZLÚ R-5904, Prague (2013), (in Czech)

6. P. Straka, Report VZLÚ R-4910, Prague (2010), (in Czech)

7. C.L. Rumsay, T.B. Gatski, Jour. Of Aircraft, 83, pp. 904-910 (2001)

8. P. Straka, M. Němec, Applied Mechanics and Materials, 821, pp. 120-128 (2016)

9. P. Straka, Proc. ECCOMAS Congress 2016, Crete, (2016) 